\title{
Chiral Mesons in Hot Matter
}

\author{
A. Gómez Nicola, F.J. LLanes-Estrada and J.R.Peláez \\ Departamentos de Física Teórica I y II, \\ Facultad de Ciencias Físicas, Universidad Complutense, 28040 Madrid, Spain
}

\begin{abstract}
We review our recent work on thermal meson properties within the Chiral Perturbation Theory framework. We will focus on the pion electromagnetic form factor, stressing its importance for Relativistic Heavy Ion Collisions. We obtain modelindependent predictions (based only on chiral symmetry) such as the temperature dependence of the pion electromagnetic charge radius. Imposing unitarity allows to describe the thermal effects of resonances such as the $\rho$ meson.
\end{abstract}

\section{Introduction}

In order to describe the meson gas formed after a Relativistic Heavy Ion Collision (RHIC) for temperatures below Chiral Symmetry Restoration, Chiral Perturbation Theory (ChPT) is the most general theoretical scheme [1] and its predictions are based only on Chiral Symmetry. When applied to the low- $T$ meson gas, one can analyze static quantities such as the pressure [2, 3, quark condensate 1, 2, 3] and pion decay constants [1, 4. All these quantities point towards Chiral Symmetry Restoration. In addition, the analysis of the pion dispersion relation $p^{2}=m_{\pi}^{2}+g\left(p_{0},|\vec{p}| ; T\right)$ shows that to leading order $O\left(T^{2}\right), g$ is real and depends only on temperature but not on momenta [1] while corrections to the pion width start to $O\left(T^{4}\right)$ [4, 15$]$.

Recently, we have shown that ChPT is equally useful to describe dynamical quantities such as scattering amplitudes [6] or form factors [7]. Furthermore, combining Chiral Symmetry Breaking and Unitarity provides a fruitful approach to describe the thermal behaviour of resonances [8] such as the $\rho$, whose medium properties are crucial to explain the excess observed in the dilepton spectrum data [9], which has motivated a huge amount of theoretical work devoted to explain in-medium resonances [10].

\section{Pion form factors at finite temperature}

The main contribution to the dilepton rate comes from the pion annihilation channel via a virtual photon exchange $\pi^{+} \pi^{-} \rightarrow \gamma^{*} \rightarrow e^{+} e^{-}$. The dilepton rate is then proportional to the pion electromagnetic form factor [11] which for $T \neq 0$ has the following general form:

$$
\begin{aligned}
\left\langle\pi^{+}(p) \pi^{-}\left(p^{\prime}\right)\left|V_{0}(0)\right| 0\right\rangle & =q_{0} F_{t}\left(S_{0},|\vec{S}|, q_{0} ; T\right) \\
\left\langle\pi^{+}(p) \pi^{-}\left(p^{\prime}\right)\left|V_{k}(0)\right| 0\right\rangle & =q_{k} F_{s}\left(S_{0},|\vec{S}|, q_{0} ; T\right)+S_{k} q_{0} G_{s}\left(S_{0},|\vec{S}|, q_{0} ; T\right)
\end{aligned}
$$




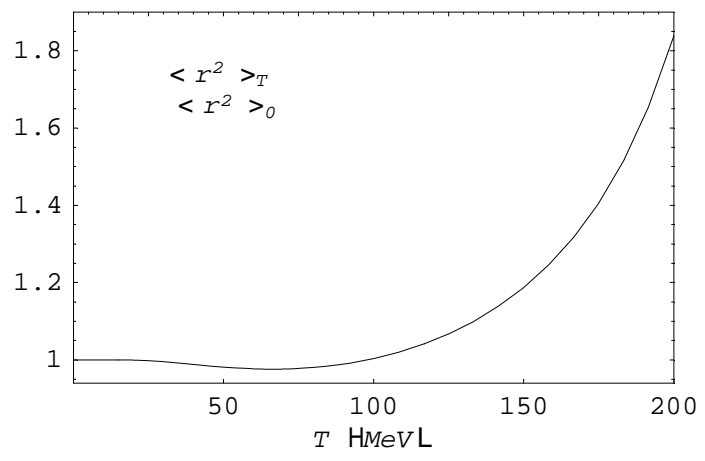

Figure 1: The pion electromagnetic charge radius at finite temperature

with $V_{\mu}$ the electromagnetic current, $S=p+p^{\prime}, q=p-p^{\prime}$ and $F_{t}, F_{s}, G_{s}$ even functions in $q_{0}$ by charge conjugation invariance. These functions are related by the gauge invariance Ward identity $\left\langle\pi \pi\left|\partial_{\mu} V^{\mu}\right| 0\right\rangle=0$.

We have calculated the one-loop ChPT form factors, whose detailed results can be found in [7]. This allows for model-independent predictions. For instance, defining the pion electromagnetic charge and radius as:

$$
\begin{aligned}
Q_{T} & =\lim _{|\vec{S}| \rightarrow 0^{+}} F_{t}(0,|\vec{S}| ; T) \\
\left\langle r^{2}\right\rangle_{T} & =-\left.\frac{6}{Q_{T}} \frac{d F_{t}(0,|\vec{S}| ; T)}{d|\vec{S}|^{2}}\right|_{|\vec{S}|=0}
\end{aligned}
$$

which takes into account Lorentz covariance breaking at $T \neq 0$, one finds the result shown in Figure 1. The radius remains almost constant for low $T$, which is consistent with the Vector Meson Dominance (VMD) idea that $\left\langle r^{2}\right\rangle \sim M_{\rho}^{-2}$ [13], provided $M_{\rho}$ changes little at low temperatures. On the other hand, the radius increases significantly for higher temperatures, dominated by a $Q_{T}$ reduction (charge screening). Estimating the critical temperature of deconfinement by requiring that the electromagnetic pion volume $V(T)=(4 \pi / 3)\left\langle r^{2}\right\rangle_{T}^{3 / 2}$ equals the inverse pion density gives $T_{c} \simeq 200 \mathrm{MeV}$, very close to the prediction of ChPT for chiral symmetry restoration [1, 2, 3]. This gives support to the idea that both transitions take place nearly at the same point. Our results for the pion charge radius confirm those in 14 based on QCD sum rules.

\section{Thermal unitarity and resonances}

In the center of mass frame $\left(\vec{p}=-\overrightarrow{p^{\prime}}\right.$, corresponding to back to back dilepton pairs) the ChPT pion form factor satisfies the following perturbative unitarity relation for energies above the two-pion threshold $S_{0}>2 m_{\pi}$ :

$$
\operatorname{Im} F^{(1)}\left(S_{0}+i \epsilon ; T\right)=\sigma_{T}\left(S_{0}\right) a_{11}^{(0)}\left(S_{0}^{2}\right)\left[F^{(0)}\left(S_{0}^{2}\right)\right]^{*}
$$

with:

$$
\sigma_{T}\left(S_{0}\right)=\sqrt{1-\frac{4 m_{\pi}^{2}}{S_{0}^{2}}}\left[1+\frac{2}{e^{S_{0} / 2 T}-1}\right]
$$


The superscripts (0) and (1) indicate the LO and NLO ChPT contributions respectively and $a_{I J}$ denote pion scattering partial waves with definite isospin $I$ and angular momentum $J$. Note that $F^{(0)}=1$ but we have displayed it in the above equation for consistency with what follows. Here, $\sigma_{T}$ is the thermal two-particle phase space, which can be understood by writing it as $\sigma_{T}=\sigma_{0}\left[\left(1+n_{B}\right)\left(1+n_{B}\right)-n_{B}^{2}\right]$ where $n_{B}=\left[\exp \left(S_{0} / 2 T\right)-1\right]^{-1}$ is the Bose-Einstein distribution function for each pion. Hence, the phase space is enhanced at finite temperature due to the difference between induced emission and absorption processes in the thermal bath [15]. This effect contributes to the resonance thermal width enhancement [8]. The partial waves $a_{I J}$ also satisfy a thermal perturbative unitarity relation [6] which is the $T=0$ one with $\sigma_{0}$ replaced by $\sigma_{T}$, as in (3).

Thermal perturbative unitarity is the key point to describe resonances within ChPT. In fact, inspired by the success of the so called Inverse Amplitude Method (IAM) at $T=0$ [16 we have constructed unitarized partial waves and form factors demanding exact thermal unitarity. For instance, $\operatorname{Im} F^{I A M}=\sigma_{T} a_{11}^{I A M}\left[F^{I A M}\right]^{*}$. The remaining freedom is fixed by matching the ChPT series at low energies. This approximation is valid for small pion densities $n_{B}$, which is very reasonable for the temperatures and energies we are interested in. The IAM generates the $\sigma(I=J=0)$ and $\rho(I=J=1)$ poles in the energy complex plane and their thermal behaviour is the expected one. In Figure 2 we have plotted the results for the poles and for the unitarized form factor. The $\rho$ widens in the thermal bath, effect also visible in the form factor, peaked at the $\rho$ mass. Such widening is in perfect agreement with the expectations from dilepton experiments and previous analysis [10, 12. Our resulting $M_{\rho}$ remains almost constant for $T<100 \mathrm{MeV}$ (consistently with VMD) and decreases slightly for higher $T$. This result points in the same direction as recent measurements by the STAR collaboration [17] but we have ignored other medium effects such as baryon density. The in-medium $\rho$ mass remains certainly an open issue [10]. As for the $\sigma$ pole, the results in Figure 2 show a behaviour compatible with Chiral Symmetry Restoration: $m_{\sigma}$ decreases sharply, hence approaching $m_{\pi}$ which increases softly with $T$ [1. The $\sigma$ width increases at first by phase space enhancement but as $m_{\sigma} \rightarrow 2 m_{\pi}$ the decay $\sigma \rightarrow 2 \pi$ is reduced and so does the width. A similar behaviour has been found in [18.
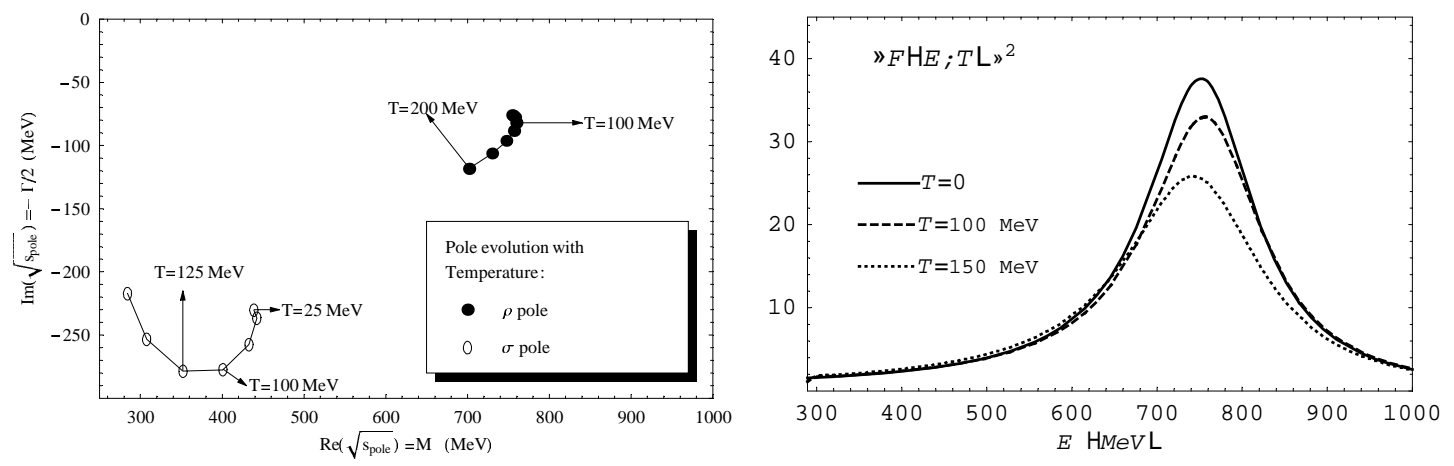

Figure 2: Thermal poles (left pannel) and unitarized form factor (right pannel).

Summarizing, ChPT and its unitarization programme can be extended successfully 
to finite temperature and provide a promising framework for future applications in the context of Relativistic Heavy Ion Collisions.

\section{Acknowledgments}

This work is supported by the Spanish research projects FPA2000-0956,PB98-0782, BFM20001326 and FPA2004-02602.

\section{References}

[1] J. Gasser and H. Leutwyler, Phys.Lett. B184, 83 (1987).

[2] P.Gerber and H.Leutwyler, Nucl.Phys.B321, 387 (1989).

[3] A.Dobado, and J.R.Peláez, Phys.Rev D59, 034004 (1999); J.R.Peláez, Phys.Rev D66, 096007 (2002).

[4] R.D.Pisarski and M.Tytgat, Phys.Rev. D54, 2989 (1996). D.Toublan, Phys.Rev. D56, 5629 (1997). J.M.Martinez Resco and M.A.Valle Basagoiti, Phys.Rev. D58, 097901 (1998).

[5] J.L. Goity and H.Leutwyler Phys.Lett. B228, 517 (1989).

[6] A. Gómez Nicola, F. J. Llanes-Estrada and J. R. Peláez, Phys.Lett. B550, 55 (2002).

[7] A. Gómez Nicola, F. J. Llanes-Estrada and J. R. Peláez, hep-ph/0405273.

[8] A. Dobado, A. Gómez Nicola, F. Llanes-Estrada and J. R. Peláez, Phys.Rev. C66, 055201 (2002).

[9] G.Agakichiev et al [CERES Collaboration] Phys. Rev. Lett. 75, 1272 (1995); Phys.Lett. B442, 405 (1998); Nucl.Phys. A 661, 23 (1999); D.Adamova et al [CERES/NA45 Collaboration], Phys. Rev. Lett., 91042301 (2003).

[10] M.Dey, V.L.Eletsky and B.L.Ioffe, Phys.Lett. B252, 620 (1990); R.D.Pisarski, Phys.Rev. D52, 3773 (1995); G. Q. Li, C. M. Ko and G. E. Brown, Phys.Rev.Lett. 75, 4007 (1995). V. Koch and C. Song, Phys. Rev. C54, 1903 (1996). R.Rapp and J.Wambach, Adv.Nucl.Phys 25:1 (2000); V. L. Eletsky, M. Belkacem, P. J. Ellis and J. I. Kapusta, Phys. Rev. C64, 035202 (2001). H.-J. Schulze and D.Blaschke, Phys.Lett. B386, 429 (1996); Part.Nucl.Lett. 119, 27 (2004).

[11] K.Kajantie, J.Kapusta, L.McLerran and A.Mekjian, Phys.Rev. D34, 2746 (1986). C.Gale and J.I.Kapusta, Phys.Rev. C35, 2107 (1987)

[12] C.Song and V.Koch, Phys.Rev. C54 3218 (1996). 
[13] J.F.Donoghue, C.Ramirez and G.Valencia, Phys.Rev. D39, 1947 (1989); G. Ecker, J. Gasser, A. Pich and E. de Rafael, Nucl.Phys. B321, 311 (1989).

[14] C. A. Dominguez, M. Loewe and J. S. Rozowsky, Phys.Lett. B335, 506 (1994).

[15] H. A. Weldon, Ann. Phys. 214, 152 (1992).

[16] T. N. Truong, Phys.Rev.Lett.61, 2526 (1988); A. Dobado, M.J.Herrero and T.N. Truong, Phys.Lett. B235, 134 (1990); A. Dobado and J.R. Peláez, Phys.Rev.D47, 4883 (1993); Phys.Rev.D56, 3057 (1997); A.Dobado, M.J.Herrero, J.R.Peláez and E.Ruiz Morales, Phys.Rev. D62, 055011 (2000); A.Gómez Nicola and J.R.Peláez, Phys.Rev.D65:054009 (2002).

[17] J. Adams et al. [STAR Collaboration], Phys.Rev. Lett. 92:092301 (2004).

[18] S.Chiku and T.Hatsuda, Phys.Rev.D57, R6 (1998); Phys.Rev.D58, 076001 (1998). K.Yokokawa, T.Hatsuda, A.Hayashigaki and T.Kunihiro, Phys.Rev. C66, 022201 (2002). 